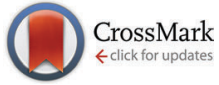

Cite this: J. Mater. Chem. C, 2017, 5, 1724

Received 6th December 2016, Accepted 16th January 2017

DOI: $10.1039 / c 6 t c 05285 a$

rsc.li/materials-c

\title{
Two-photon absorption in penicillamine capped CdS tetrapods
}

\author{
D. Wawrzyńczyk
}

\begin{abstract}
The two-photon absorption of water soluble CdS tetrapods, stabilized with a racemic mixture of chiral ligands, was measured using two techniques: open-aperture Z-scan and two-photon excited emission. The investigated CdS tetrapods showed two-photon absorption in the wavelength range 575-875 $\mathrm{nm}$, with the maximum value of $7900 \mathrm{GM}$ at $625 \mathrm{~nm}$. Thanks to the high luminescence quantum yield, and relatively strong two-photon absorption, the CdS tetrapods showed intense two-photon excited emission upon near-infrared excitation with two-photon excitation action cross-section equal to $3200 \mathrm{GM}$ and $420 \mathrm{GM}$ at $650 \mathrm{~nm}$ and $700 \mathrm{~nm}$, respectively. An evident blue shift of the two-photon absorption maxima with respect to the one-photon spectra at the wavelengths multiplied by two was observed, and discussed based on the reported theoretical calculation for CdS quantum dots with tetrahedral symmetry. Since CdS tetrapod arms show low electron and hole densities, they do not contribute significantly to the values of the two-photon absorption cross-section. The performed investigation of the optical properties of these water soluble colloidal nanoparticles indicates their potential applications in nonlinear bioimaging.
\end{abstract}

\section{Introduction}

The ligand or shape induced chirality occurring in inorganic nanostructures has recently become a new emerging field in materials science and nanophotonics. ${ }^{1,2}$ The optical activity, in e.g. metallic nanostructures of semiconducting quantum dots (QDs), can be induced by (i) chiral crystal structure dislocations spontaneously occurring during the growth process, ${ }^{3,4}$ (ii) shape directed synthesis ${ }^{1,5}$ or (iii) post-synthetic surface treatment. ${ }^{6}$ In particular, II-VI type semiconducting QDs functionalized with chiral molecules, e.g. cysteine ${ }^{6-8}$ penicillamine $^{9-12}$ or glutathione, ${ }^{13-15}$ have gained increasing attention due to the possibility of size-tuning of their chiro-optical activity. Organic ligands used for QD surface functionalization induced chiral distortion of surface atoms, ${ }^{10,16}$ and thus the appearance of additional circular dichroism (CD) bands in the wavelength ranges associated with QD electronic levels. Most commonly used chiral ligands contain sulfur atoms with high affinity for the cadmium element of CdSe, CdS or CdTe QDs, which further facilitates the post-synthetic ligand exchange processes. After surface functionalization with chiral molecules the QDs can be transferred to a non-toxic water environment, which allows for their application in bio-imaging ${ }^{8}$ or chiral drug molecular recognition. ${ }^{7}$ S. Tamang et al. ${ }^{17}$ reported detailed optimization

Advanced Materials Engineering and Modelling Group, Faculty of Chemistry, Wrocław University of Technology, Wybrzeże Wyspiańskiego 27, 50-370 Wroclaw, Poland. E-mail: dominika.wawrzynczyk@pwr.edu.pl of aqueous phase transfer of various types of QDs with the use of cysteine molecules, after which the QDs preserved high quantum yield (QY) and prolonged colloidal stability. In another approach, chiral surface stabilizers could be used directly during the synthesis process, as growth controlling factors, which was shown for CdS tetrapods. ${ }^{18,19}$ Such an approach allows omission of the surface functionalization step, which usually causes unwanted material loss, and additionally enables a significant reduction of the reaction temperature from above $300{ }^{\circ} \mathrm{C}$ down to $100-150{ }^{\circ} \mathrm{C}$. CdSe and CdS semiconducting QDs are also known as good bio-imaging markers because of their optical and chemical stability, resistance to photobleaching, as well as strong twophoton absorption (TPA) and intense two-photon excited emission (TPEE)..$^{20,21}$ The use of near-infrared two-photon excitation decreases the autofluorescence background signal and increases image resolution. Martynenko et $a .^{8}{ }^{8}$ recently showed the enantioselective cellular uptake of $\mathrm{L}^{-}$and $\mathrm{D}^{-}$-cysteine functionalized CdSe/ZnS core/shell QDs, while Li et al. ${ }^{13}$ showed chirality dependent cytotoxicity and cellular metabolism of $\mathrm{L}^{-}$and D-glutathione capped CdTe QDs. Thus the combination of decreased toxicity, chiral selectivity and large TPA can open new perspectives for surface engineered semiconducting QDs applications. An important issue for finding the optimum excitation conditions for the two-photon excited emission of such systems is a detailed investigation of the wavelength dependence of TPA cross-sections $\left(\sigma_{2}\right)$ and TPEE intensity in chiral QDs. While in the case of chiral functionalized, spherical QDs neither the position of the TPA maximum nor the $\sigma_{2}$ values should differ 
significantly from those measured for the QDs with a similar radius ${ }^{20,22}$ the situation is rather more ambiguous for tetrapodlike shaped CdS QDs. Due to the symmetry breaking or change in the oscillator strength of certain two-photon allowed transitions, the position of TPA maxima can be shifted with respect to exciton one-photon absorption (as plotted against the wavelength simply multiplied by two), additionally the reduced symmetry in quantum confined systems is known to increase the density of states and thus increase the TPA cross-section values. ${ }^{23}$

Here, two different femtosecond laser-based techniques, i.e. open-aperture (OA) Z-scan ${ }^{24,25}$ and comparison of TPEE emission intensity of CdS tetrapods with that of fluorescein dye, ${ }^{26}$ were used to determine the wavelength dependence of $\sigma_{2}$ values for water soluble CdS tetrapods synthesized in the presence of a racemic mixture of penicillamine molecules. The obtained results were further confronted with the corresponding data measured previously for spherical CdS QDs,${ }^{27}$ and discussed in view of the possible application of CdS tetrapods as markers in nonlinear bio-imaging.

\section{Experimental}

The CdS tetrapods were synthesized based on protocols described previously, ${ }^{18,19}$ with the use of a racemic mixture of L- and D-penicillamine molecules as a stabilizing agent. The deliberate choice of such a ligand composition, allowed the treatment of the synthesized CdS tetrapods as, to some extent, a reference sample. Changing between left- and right-handed enantiomers of penicillamine used as stabilizing ligands for the synthesis of CdS tetrapods should neither significantly influence their morphology nor their linear optical properties, i.e. the position of the absorption and emission maxima, lifetime or quantum yield. ${ }^{18,19}$ Due to the chosen ligands, the reaction could be performed in a nontoxic water environment at a relatively low temperature. Briefly, $10 \mathrm{ml}$ of $0.01 \mathrm{M}$ water solution $(\mathrm{pH} \sim 11$ stabilized with $2 \mathrm{M} \mathrm{NaOH})$ of penicillamine (50:50 mixture of $\mathrm{L}$ - and $\mathrm{D}$-enantiomer) was mixed in a three neck flask with $8 \mathrm{ml}$ of $\mathrm{CdCl}_{2}$ solution $(0.01 \mathrm{M})$ and $2 \mathrm{ml}$ of thioacetamide $(0.01 \mathrm{M})$, followed by the addition of $40 \mathrm{ml}$ of distilled water. The reaction mixture was heated to $130{ }^{\circ} \mathrm{C}$ under reflux, with magnetic stirring, and maintained under those conditions for $2 \mathrm{~h}$, followed by aging in the dark at room temperature overnight. Next, the rotary evaporator (BUCHI) was used to reduce the volume of the obtained yellow CdS tetrapod solution to $\sim 2 \mathrm{ml}$. The sample was purified by centrifugation $(2 \mathrm{~min}$, $1800 \mathrm{rpm}$ ) and additionally by passing through $30 \mathrm{kDa}$ centrifuge filters (10 $\mathrm{min}, 1800 \mathrm{rpm})$. The morphology of the obtained CdS tetrapods was investigated using a FEI Tecnai $\mathrm{G}^{2} 20$ X-TWIN transmission electron microscope (TEM). The absorption and emission spectra of dilute CdS tetrapod water solution were measured using a JASCO V670 and a Shimadzu HITACHI UV-3600 spectrometers, respectively. The luminescence lifetime $(\tau)$ was measured using a time-correlated single-photon counting (TCSPC) self-constructed setup based on Becker\&Hickl hardware, consisting of a data acquisition module (SPC-130-EM) and a hybrid PMT detector (HPM-100-06) mounted onto a Priceton Instruments spectrograph (Acton SpectraPro-2300i) under excitation with a $375 \mathrm{~nm}$ picosecond laser diode (BDL-375-SMC). The dedicated Becker\&Hickl SPCImage software was used for the $\tau$ value calculation. An FLS 980 spectrophotometer (Edinburgh Instruments), with a dedicated integrating sphere and FLS software, was used to determine the QY of the investigated CdS tetrapod sample. As a reference the cuvette field with water was used to extract the unwanted contribution of light scattering. Finally, two independent techniques, i.e. open aperture (OA) Z-scan ${ }^{25}$ and two-photon excited emission (TPEE), ${ }^{26}$ were used for the determination of wavelength dependence of $\sigma_{2}$ values, both using a laser system consisting of a Quantronix Integra-C Ti:sapphire regenerative amplifier, which produces $\sim 130 \mathrm{fs}$, $800 \mathrm{~nm}$ pulses with $1 \mathrm{kHz}$ repetition rate, and $1 \mathrm{~mJ}$ energy per pulse, and a Quantronix Palitra-FS optical parametric amplifier allowing for wavelength tuning between 530 and $2000 \mathrm{~nm}$. An OceanOptics USB2000 fiber spectrophotometer was used for acquiring the two-photon excited spectra of CdS tetrapods and a reference fluorescein solution. The detailed description of both experimental setups can be found in previous papers. ${ }^{24,28}$

\section{Results and discussion}

Investigated CdS tetrapods were water soluble directly after synthesis, and no post-synthetic surface treatment was necessary for transferring those nanoparticles to a nontoxic environment, which further facilitates their application as optical markers in bio-imaging. Fig. 1a and b show TEM images of synthesized CdS tetrapods, and a high resolution inset in Fig. 1b presents lattice fringes, which proves high crystallinity of the synthesized materials. Based on the TEM images, and measuring the sizes of over 100 particles (see Fig. 1c for size distribution histograms) the length and width of CdS tetrapod arms were calculated to be $13.65 \pm 0.27 \mathrm{~nm}$ and $4.86 \pm 0.14 \mathrm{~nm}$, respectively. Due to the large difference between the central core size and the arm's length one could expect the efficient quantum confinement inside the tetrahedron-like CdS core. In fact, theoretical calculations for similar $\mathrm{CdSe}^{29}$ and $\mathrm{CdTe}^{30}$ tetrapods have shown the localization of the lowest electronic states inside the central tetrahedron, while the first hole state slightly enters the arms of the tetrapod. The wave function of the lowest twenty electron and hole states, which govern the optical properties of tetrapodlike shaped semiconducting nanoparticles, was also found to be strongly confined to a small central-core region. ${ }^{31}$ The dimensions of CdS tetrapods, and the density of bulk CdS $\left(4.83 \mathrm{~g} \mathrm{~cm}^{-3}\right)$, were used to estimate the molecular weight (MW) of a single particle. The volume of a single CdS tetrapod was calculated as a sum of the volume of the tetrahedron core and four pyramid arms. The inset in Fig. 1d shows the 3D model of the CdS tetrapod structure. Based on the above assumptions the MW of a single CdS tetrapod was calculated to be equal to $577600 \mathrm{~g} \mathrm{~mol}^{-1}$. Fig. $1 \mathrm{~d}$ presents the absorption and emission spectra of dilute water solution of CdS tetrapods, which showed similar optical features to those previously reported for such nanostructures. ${ }^{18,19}$ The lowest 

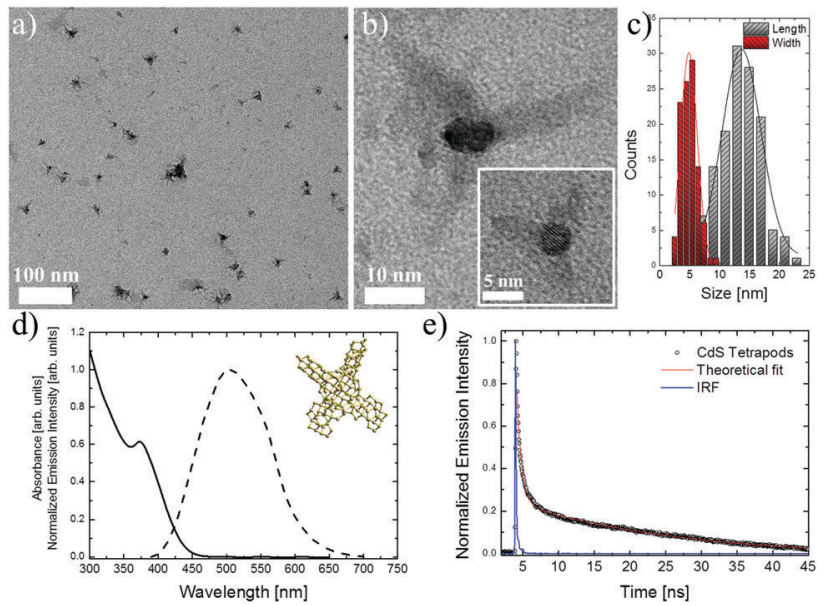

e)

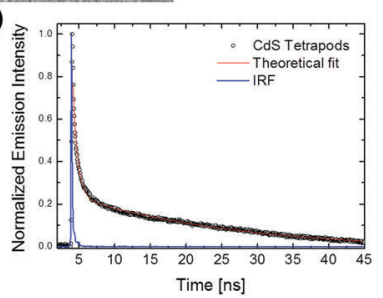

Fig. 1 TEM images of the synthesized CdS tetrapods ( $a$ and $b$ ), the inset in (b) shows a high-resolution image with clearly visible lattice fringes, with arm's length and width size distribution histograms (c) calculated based on measuring the sizes of over 100 particles. Absorption (solid line) and normalized emission (dashed) spectra under $375 \mathrm{~nm}$ excitation (d). Inset in (d) shows a CdS tetrapod structure model. A fluorescence decay curve, together with double exponential fitting, of CdS tetrapods measured with a time correlated single photon counting technique upon $375 \mathrm{~nm}$ picosecond laser diode excitation (e).

exciton absorption peak was situated at approximately $375 \mathrm{~nm}$, and under $375 \mathrm{~nm}$ excitation the CdS tetrapods showed broad band emission between $400 \mathrm{~nm}$ and $700 \mathrm{~nm}$ with maximum at $\sim 500 \mathrm{~nm}$. The significant broadening of the emission band, when compared to spherical CdS QDs, ${ }^{27,32}$ can arise in general from two reasons: (i) large contribution of the defects or trapped states on the surface of nanoparticles, or (ii) due to the distribution of the particle sizes in the solution, ${ }^{11}$ and the distinction between those two can be made based on QY and emission kinetic measurements. The luminescence lifetime of CdS tetrapod emission showed a double exponential character, and the values of the long and short components of luminescence lifetime were calculated, after deconvolution of the instrument response function (IRF), to be $\tau_{1}=29.75 \pm 0.61 \mathrm{~ns}$ and $\tau_{2}=0.947 \pm 0.010 \mathrm{~ns}$, respectively (Fig. 1e). While the QY of the investigated CdS tetrapods was determined to be at the level of $\sim 60 \%$. The observed short luminescence lifetime, with a simultaneous high QY value, suggests the near band edge character of the observed emission rather than the defect related one, which would in fact decrease the QY value and increase the decay time. The synthesis of CdS based quantum confined nanostructures is a much slower process, taking hours rather than seconds or minutes, when compared to e.g. synthesis of CdSe QDs. ${ }^{22,33}$ The slow reaction kinetics assures higher quality of the obtained nanomaterials with a large number of radiative surface-states and low nonradiative surface quenching defects. ${ }^{34}$ The high QY and short $\tau$ also suggest that the observed emission band broadening is likely due to the CdS tetrapod size distribution, which can be in fact seen in the TEM images and size distribution histograms (Fig. 1a and c). The observed intense emission, with a relatively high QY, of the synthesized CdS tetrapods is a very desirable feature for optical staining agents. The main drawback relates, however, to the necessity of using blue or ultraviolet light excitation, but that could be overcome by two-photon, nearinfrared excitation.

The two-photon absorption cross-section values of the investigated CdS tetrapods were first calculated based on the comparison of the TPEE intensity of the commercially available fluorescein dye $\left(3.4 \times 10^{-9} \mathrm{~mol} \mathrm{~cm}^{-3}\right.$ in $\left.0.1 \mathrm{M} \mathrm{NaOH}, \mathrm{QY}=91 \%\right)$ with that of the CdS tetrapods dispersed in water $\left(3.9 \times 10^{-9} \mathrm{~mol} \mathrm{~cm}{ }^{-3}\right)$ using the below equation, and the $\sigma_{2 \text {,Fluo. values for fluorescein }}$ measured by Makarov et al.: ${ }^{26}$

$$
\sigma_{2, \text { CdSTetra. }}=\frac{F_{2, \text { CdSTetra. }} C_{\text {Fluo. }} \text { QY }}{F_{2, \text { Fluo. }} C_{\text {CdS Tetra. }} \text { QY }} \sigma_{\text {CdS Tetra. }} \sigma_{2, \text { Fluo. }}
$$

where $F$ is the fluorescence intensity and $C$ is the sample concentration. A similar technique was previously used for the determination of $\sigma_{2}$ values of InP@ZnS core@shell QDs of different sizes. ${ }^{28}$ As in that case, to assure the two-photon character of the observed CdS tetrapods emission upon near-infrared excitation, measurements of power dependence of emission integral intensities under $750 \mathrm{~nm}$ excitation were performed. Fig. 2a shows the CdS tetrapod emission spectra measured upon changing the laser excitation power (from $100 \mathrm{~mW}$ up to $300 \mathrm{~mW}$ average power), and Fig. $2 \mathrm{~b}$ presents the corresponding double logarithmic plot of the integral emission intensities $v s$. incident beam power. The linear fitting of the data points resulted in the slope value equal to 2.11, which proves the two-photon character of the observed process. Using the TPEE technique it was possible to measure the $\sigma_{2}$ values in the range of 700-900 nm (Fig. 2c black circles), above $875 \mathrm{~nm}$ no fluorescence emission from the CdS tetrapods was detected, while shorter wavelength excitation would interfere with the collection of the fluorescence signal.

The second method used for the determination of $\sigma_{2}$ values was the OA Z-scan technique introduced by Sheik-Bahae et al. ${ }^{25}$ A concentrated CdS tetrapod $\left(116.2 \times 10^{-9} \mathrm{~mol} \mathrm{~cm}^{-3}\right)$ water solution was placed in a $1 \mathrm{~mm}$ glass cuvette, and its transmittance was recorded as the sample traveled along a focused femtosecond laser beam. For the determination of the incident light intensity, and for assuring that no contributions of cuvette cells and solvent to the OA signal existed, the measurements were first performed for a fused silica plate $(4.6 \mathrm{~mm}$ thick) and for the cuvette filled with water alone, respectively. The OA Z-scan measurements were performed for the wavelengths from $575 \mathrm{~nm}$ to $850 \mathrm{~nm}$; however, above $725 \mathrm{~nm}$ no distinguishable dips in the OA raw Z-scan traces were detected. It needs to be remarked that, since Z-scan measurements detect changes in the transmittance that may be relatively small, they are much more susceptible than the TPEE technique to the noise resulting from laser intensity fluctuations.

The comparison of the $\sigma_{2}$ values calculated based on the fluorescence (black circles) and Z-scan methods (red squares) is presented in Fig. 2c, together with the linear absorption spectra plotted against the doubled wavelength (grey area). The maximum values of TPA were observed in the short wavelength range from $575 \mathrm{~nm}$ to $675 \mathrm{~nm}$, peaking at $625 \mathrm{~nm}$ with $\sigma_{2}=7900 \mathrm{GM}$ $\left(1 \mathrm{GM}=10^{-50} \mathrm{~cm}^{4} \mathrm{~s}^{-1}\right.$ photon $\left.{ }^{-1}\right)$. For the wavelengths above 

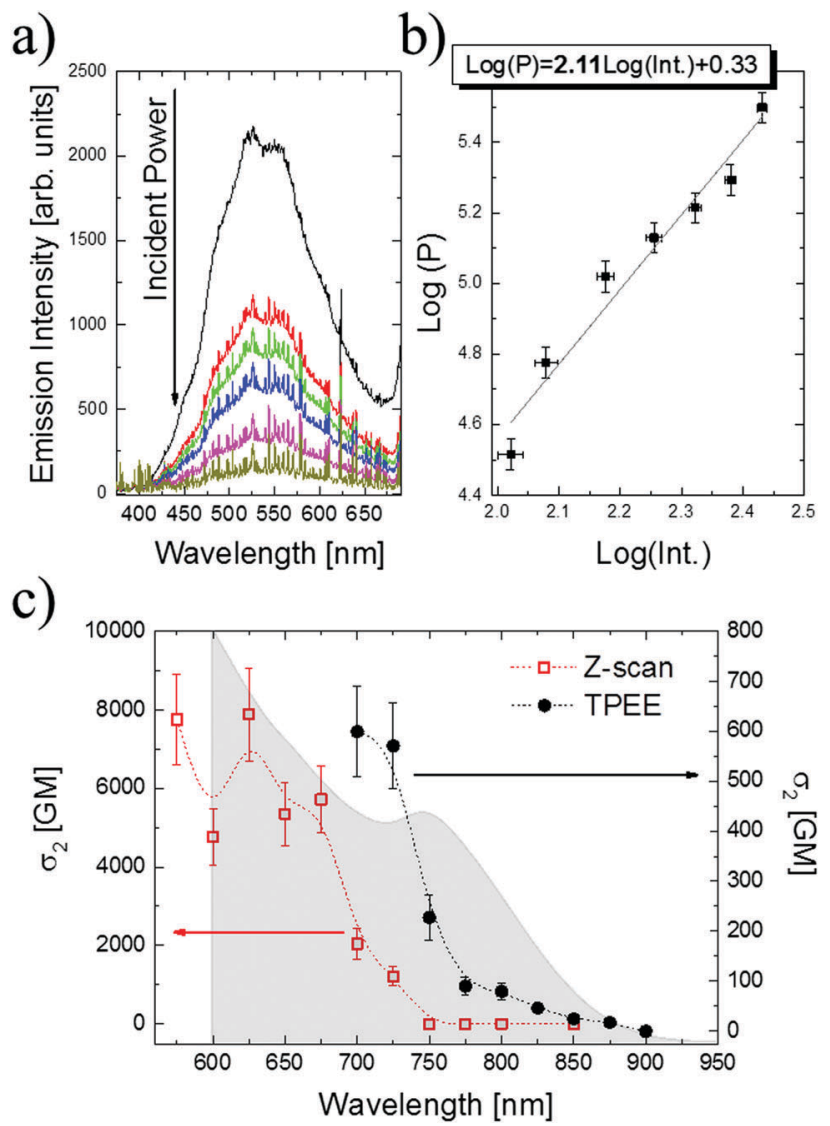

Fig. 2 TPEE spectra of CdS tetrapods measured for various laser powers (a), together with a double logarithmic plot of the emission integral intensity vs. $750 \mathrm{~nm}$ femtosecond laser power fitted with the linear function with the slope equal to 2.11 (b). Wavelength dispersion of TPA cross-section, $\sigma_{2}$ taken per individual CdS tetrapod, measured by TPEE (black circles) and Z-scan (red squares) techniques (c), with comparison to one-photon absorption spectra plotted against wavelength multiplied by two (grey area). The lines in the $\sigma_{2}$ plots were added just as the guide to the eye.

$725 \mathrm{~nm}$ the Z-scan measurements did not detect evident TPA signals; however, the upconverted green emission from CdS tetrapods was still visible when the sample passed through the focal plane of the lens. This characteristic emission could be in fact observed upon excitation with laser wavelengths up to $900 \mathrm{~nm}$, and based on TPEE measurements the $\sigma_{2}$ values for longer wavelengths were calculated to reach $600 \mathrm{GM}$ at $700 \mathrm{~nm}$. Considering the maximum value of $\sigma_{2}$ obtained from Z-scan at $625 \mathrm{~nm}$, the molecular weight $\left(\sigma_{2} / \mathrm{MW}\right)$ and volume $\left(\sigma_{2} / \mathrm{V}\right)$ scaled TPA cross-section values are equal to 0.014 (in GM mol g ${ }^{-1}$ ) and 39 (in $\mathrm{GM} \mathrm{nm}^{-3}$ ), respectively.

Based on the analysis of the $\sigma_{2}$ values of wavelength dispersion (Fig. 2c) two important features could be noticed: (i) firstly, the maximum of TPA cross-section appears to be significantly blue shifted $(\sim 125 \mathrm{~nm}, \sim 0.33 \mathrm{eV})$ with respect to the first exciton peak in the one-photon absorption spectra multiplied by two (Fig. 2c grey area) and also with respect to the TPA maxima measured previously for spherical CdS QDs; ${ }^{27,35}$ (ii) secondly, the peak $\sigma_{2}$ values as well as the scaled parameter values $\left(\sigma_{2} / \mathrm{MW}\right.$ and $\left.\sigma_{2} / \mathrm{V}\right)$ are not noticeably enhanced with respect to the corresponding ones measured using the same experimental setup for spherical CdS QDs, ${ }^{27}$ as well as with those reported in the literature (Table 1 ). Table 1 provides the $\sigma_{2}$ values reported in the literature together with molecular weight and nanoparticle volume scaled parameters measured for spherical CdS QDs, as well as for elongated CdS quantum rods (QRs). It should be noticed that the investigated CdS tetrapods show no axial or spherical symmetry; additionally, their size can be considered large when compared to the exciton Bohr radius for CdS materials $(5.8 \mathrm{~nm}) .{ }^{36,37}$ The anisotropic shape of CdS or CdSe QRs is known to greatly enhance the TPA strength (for values and references see Table 1) due to the reduced symmetry and increased density of states (DOS) by anisotropy splitting of degenerate energy levels in cylindrical nanomaterials. ${ }^{23,35}$ Allione et $a .^{38}$ experimentally and theoretically ( $k \cdot p$ model calculations) investigated the nonlinear properties of $\mathrm{CdSe} / \mathrm{CdS}$ dot-in-rod heterostructures, with an even lower degree of symmetry than QRs. Those authors also observed an enhancement of 2PA crosssection for QRs when compared to QDs, with a simultaneous blue shift ( $85 \mathrm{~nm})$ of the TPA maxima relative to the one-photon transitions. ${ }^{38}$ However, no evidence for the selection rule violations due to symmetry breaking was found. Shifting of the TPA maxima towards higher energy was concluded to be a consequence of different selection rules for one- and two-photon

Table 1 Comparison of values of the 2PA cross-section $\left(\sigma_{2}\right)$, molecular weight $\left(\sigma_{2} / \mathrm{MW}\right)$ and nanoparticle volume $\left(\sigma_{2} / \mathrm{V}\right)$ scaled parameters measured for CdS QDs and QRs using different techniques

\begin{tabular}{|c|c|c|c|c|c|c|}
\hline CdS sample (size) & $\begin{array}{l}\lambda_{\text {exc. }} \\
{[\mathrm{nm}]}\end{array}$ & $\begin{array}{l}\sigma_{2} \\
{\left[\mathrm{GM}=10^{-50} \mathrm{~cm}^{4} \mathrm{~s}^{-1} \text { photon }^{-1}\right]}\end{array}$ & $\begin{array}{l}\sigma_{2} / \mathrm{MW} \\
{\left[\mathrm{GM} \mathrm{mol} \mathrm{g}^{-1}\right]}\end{array}$ & $\begin{array}{l}\sigma_{2} / \mathrm{V} \\
{\left[\mathrm{GM} \mathrm{nm}^{-3}\right]}\end{array}$ & Technique & Ref. \\
\hline QDs $(4.45 \mathrm{~nm})$ & 800 & 4000 & 0.030 & 86 & Z-scan & 40 \\
\hline QDs $(4.7 \mathrm{~nm})$ & 800 & 5300 & 0.034 & 98 & Z-scan & 40 \\
\hline QDs (5.3 nm) & 800 & 8400 & 0.037 & 108 & Z-scan & 40 \\
\hline QDs $(4.1 \mathrm{~nm})$ & 800 & 7900 & 0.075 & 219 & Z-scan & 41 \\
\hline QDs (5 nm) & 750 & 7200 & 0.038 & 110 & Z-scan & 27 \\
\hline QDs (4.5 nm) & 800 & 12000 & 0.087 & 252 & Z-scan & 42 \\
\hline QDs in polymeric film $(2 \mathrm{~nm})$ & 800 & 7000 & 0.58 & 1671 & Optical Kerr effect & 43 \\
\hline QRs $(4 \times 35 \mathrm{~nm})$ & 800 & 190000 & 0.12 & 339 & Z-scan & 42 \\
\hline QRs $(4 \times 43 \mathrm{~nm})$ & 800 & 209000 & 0.11 & 304 & Z-scan & 40 \\
\hline QDs (4.5 nm) & 800 & 16000 & 0.12 & 335 & TPEE & 35 \\
\hline QRs $(5 \times 40 \mathrm{~nm})$ & 800 & 260000 & 0.090 & 260 & TPEE & 35 \\
\hline Tetrapods $(5 \times 14 \mathrm{~nm})$ & 625 & 7900 & 0.014 & 39 & Z-scan & This work \\
\hline Tetrapods $(5 \times 14 \mathrm{~nm})$ & 700 & 600 & 0.001 & 3.02 & TPEE & This work \\
\hline
\end{tabular}


transitions, and of the reduced oscillator strength of low-energy two-photon transition in $\mathrm{CdSe} / \mathrm{CdS}$ dot-in-rod heterostructures. ${ }^{38}$ The interpretation of the TPA spectral dispersion and strength measured for CdS tetrapods (Fig. 2c) can be to some extent based on the theoretical calculations reported by V. A. Fonoberov et al., ${ }^{39}$ and the calculations of individual electron + hole and exciton states in semiconducting type II-VI tetrapod-shaped nanocrystals. ${ }^{30,31}$ The former ${ }^{39}$ investigated the exciton states and optical transition in colloidal CdS QDs with spherical and tetrahedral shapes. The authors have shown that the electron and hole densities near the edges and vertices of tetrahedra are extremely small, and that it is the core of the QD that mainly contributes to the observed optical transitions. The calculations performed for quantum dots with ideal tetrahedral symmetry reviled the strong confinement of the lowest exciton into the small central spherical-core region of tetrapods. ${ }^{30}$ Additionally, it was found that in the case of CdS tetrapods the lowest excitons have optically inactive $T_{2}$ symmetry. However, due to the small energy difference between these lowest $T_{2}$ excitons and the lowest one photon optically-active $A_{1}$ excitons the room-temperature thermal energy mixes the population of these two exciton states, and luminescence from the $A_{1}$ exciton level must be observed. ${ }^{31}$ As the CdS tetrapods show a similar symmetry to tetrahedral QDs, the reduced DOS can be expected at the particle arms, which together with theoretically shown confinement of excitons inside the core region suggests that mainly the cores of CdS tetrapods contribute to the TPA strength. This is likely to be the case since the peak $\sigma_{2}$ value for CdS tetrapods is close to those measured for 4-5 nm spherical CdS QDs (Table 1). The elongated arms of the CdS tetrapods increase the MW and the volume of the particle, which on the other hand, together with low contribution to the $\sigma_{2}$ enhancement, results in a decreased molecular weight and nanoparticle volume scaled parameters, when compared to spherical QDs (Table 1). The mixing of the lowest $T_{2}$ and $A_{1}$ symmetry states in CdS tetrapods ${ }^{31}$ can be the reason for shifting the TPA maxima towards higher energy, since the linear and two-photon nonlinear processes are governed by different selection rules. Fonoberov et $a l^{39}$ also found that, in the case of tetrahedral CdS QDs, the number of optically active exciton states is much larger than for the spherical CdS QDs with high symmetry. Those transitions had low oscillator strength, however, as was also shown by Allione et al., ${ }^{38}$ the selection rules for one-and two-photon transitions are different, and thus the additional exciton states observed in tetrahedral CdS QDs can contribute to the two-photon transitions. Additionally, the exciton states in the spherical CdS QDs were higher in energy than the corresponding ones for tetrahedral QDs, ${ }^{39}$ which can also be the reason for the blue shift of the TPA spectra maxima observed for CdS tetrapods.

\section{Conclusions}

In conclusion, the investigated water soluble CdS tetrapods can be considered as interesting candidates for optical markers in nonlinear biophotonics. They showed intense emission, of near band edge character rather than the defect one, with a relatively high quantum yield. This intense emission was also observed upon near-infrared two-photon excitation, and the TPA crosssection was found to peak at $625 \mathrm{~nm}$ with $\sigma_{2}=7900 \mathrm{GM}$. The two-photon excitation action cross-section $\left(\sigma_{2}{ }^{*} \mathrm{QY}\right)$ of CdS tetrapods, the parameter relevant for nonlinear bioimaging, was equal to $3200 \mathrm{GM}$ and $420 \mathrm{GM}$ at $650 \mathrm{~nm}$ and $700 \mathrm{~nm}$, respectively. Those values are at the level of the corresponding ones reported for organic molecules designed specifically for enhanced two-photon absorption $^{44}$ and one order of magnitude lower than that for water soluble CdSe QDs. ${ }^{20}$ The tetrahedral symmetry of the investigated nanoparticles strongly influences the spectral position and the peak values of $\sigma_{2}$, and it is suggested that CdS tetrapod arms do not significantly contribute to the strength of TPA; however, it results in the shift of the TPA maxima.

\section{Acknowledgements}

The support from the Ministry of Science and Higher Education in years 2015-2017 under grant "Iuventus Plus", project no. IP2014 050273 is greatly acknowledged. DW acknowledges Ms Marcelina Chachuła for the help with the CdS tetrapod synthesis.

\section{References}

1 A. Ben-Moshe, B. Maoz, A. O. Govorov and G. Markovich, Chem. Soc. Rev., 2013, 42, 7028-7041.

2 Y. Xia, Y. Zhou and Z. Tang, Nanoscale, 2011, 3, 1374-1382.

3 A. S. Baimuratov, I. D. Rukhlenko, Y. K. Gun'ko, A. V. Baranov and A. V. Fedorov, Nano Lett., 2015, 15, 1710-1715.

4 M. V. Mukhina, V. G. Maslov, A. V. Baranov, A. V. Fedorov, A. O. Orlova, F. Purcell-Milton, J. Govan and Y. K. Gun'ko, Nano Lett., 2015, 15, 2844-2851.

5 K. T. Yong, Y. Sahoo, M. T. Swihart and P. N. Prasad, Adv. Mater., 2006, 18, 1978-1982.

6 U. Tohgha, K. K. Deol, A. G. Porter, S. G. Bartko, J. K. Choi, B. M. Leonard, K. Varga, J. Kubelka, G. Muller and M. Balaz, ACS Nano, 2013, 7, 11094-11102.

7 T. Delgado-Pérez, L. M. Bouchet, M. de la Guardia, R. E. Galian and J. Pérez-Prieto, Chem. - Eur. J., 2013, 19, 11068-11076.

8 I. V. Martynenko, V. A. Kuznetsova, I. K. Litvinov, A. O. Orlova, V. G. Maslov, A. V. Fedorov, A. Dubavik, F. Purcell-Milton, Y. K. Gun'ko and A. V. Baranov, J. Nanotechnol., 2016, 27, 075102.

9 A. Ben Moshe, D. Szwarcman and G. Markovich, ACS Nano, 2011, 5, 9034-9043.

10 S. D. Elliott, M. P. Moloney and Y. K. Gun'ko, Nano Lett., 2008, 8, 2452-2457.

11 S. A. Gallagher, M. P. Moloney, M. Wojdyla, S. J. Quinn, J. M. Kelly and Y. K. Gun'ko, J. Mater. Chem., 2010, 20, 8350-8355.

12 M. Wojdyla, S. A. Gallagher, M. P. Moloney, Y. K. Gun'ko, J. M. Kelly, L. M. Magno, S. J. Quinn, I. P. Clark, G. M. Greetham and M. Towrie, J. Phys. Chem. C, 2012, 116, 16226-16232.

13 Y. Li, Y. Zhou, H.-Y. Wang, S. Perrett, Y. Zhao, Z. Tang and G. Nie, Angew. Chem., Int. Ed., 2011, 50, 5860-5864. 
14 Y. Zhou, Z. Zhu, W. Huang, W. Liu, S. Wu, X. Liu, Y. Gao, W. Zhang and Z. Tang, Angew. Chem., Int. Ed., 2011, 50, 11456-11459.

15 Z. Zhu, J. Guo, W. Liu, Z. Li, B. Han, W. Zhang and Z. Tang, Angew. Chem., Int. Ed., 2013, 52, 13571-13575.

16 Y. L. Zhou, M. Yang, K. Sun, Z. Y. Tang and N. A. Kotov, J. Am. Chem. Soc., 2010, 132, 6006-6013.

17 S. Tamang, G. Beaune, I. Texier and P. Reiss, ACS Nano, 2011, 5, 9392-9402.

18 J. E. Govan, E. Jan, A. Querejeta, N. A. Kotov and Y. K. Gun'ko, Chem. Commun., 2010, 46, 6072-6074.

19 M. P. Moloney, J. Govan, A. Loudon, M. Mukhina and Y. K. Gun'ko, Nat. Protoc., 2015, 10, 558-573.

20 D. R. Larson, W. R. Zipfel, R. M. Williams, S. W. Clark, M. P. Bruchez, F. W. Wise and W. W. Webb, Science, 2003, 300, 1434-1436.

21 X. Michalet, F. F. Pinaud, L. A. Bentolila, J. M. Tsay, S. Doose, J. J. Li, G. Sundaresan, A. M. Wu, S. S. Gambhir and S. Weiss, Science, 2005, 307, 538-544.

22 M. Nyk, D. Wawrzynczyk, J. Szeremeta and M. Samoc, Appl. Phys. Lett., 2012, 100, 041102.

23 X. B. Feng and W. Ji, Opt. Express, 2009, 17, 13140-13150.

24 M. Nyk, D. Wawrzynczyk, K. Parjaszewski and M. Samoc, J. Phys. Chem. C, 2011, 115, 16849-16855.

25 M. Sheikbahae, A. A. Said, T. H. Wei, D. J. Hagan and E. W. Vanstryland, IEEE J. Quantum Electron., 1990, 26, 760-769.

26 N. S. Makarov, M. Drobizhev and A. Rebane, Opt. Express, 2008, 16, 4029-4047.

27 J. Szeremeta, M. Nyk, D. Wawrzynczyk and M. Samoc, Nanoscale, 2013, 5, 2388-2393.

28 D. Wawrzynczyk, J. Szeremeta, M. Samoc and M. Nyk, APL Mater., 2015, 3, 116108.

29 J. Li, Wang, Nano Lett., 2003, 3, 1357-1363.

30 K. Sakoda, Y. Z. Yao, T. Kuroda, D. N. Dirin and R. B. Vasiliev, Opt. Mater. Express, 2011, 1, 379-390.
31 Y. Z. Yao, T. Kuroda, D. N. Dirin, A. A. Irkhina, R. B. Vasiliev and K. Sakoda, Opt. Mater. Express, 2013, 3, 977-988.

32 A. Veamatahau, B. Jiang, T. Seifert, S. Makuta, K. Latham, M. Kanehara, T. Teranishi and Y. Tachibana, Phys. Chem. Chem. Phys., 2015, 17, 2850-2858.

33 X. G. Peng, L. Manna, W. D. Yang, J. Wickham, E. Scher, A. Kadavanich and A. P. Alivisatos, Nature, 2000, 404, 59-61.

34 L.-Y. Chen, C.-H. Tseng, H.-L. Chou and C.-H. Chen, Surface Modification of CdSe and CdS Quantum Dots-Experimental and Density Function Theory Investigation, 2012.

35 A. W. Achtstein, A. Ballester, J. L. Movilla, J. Hennig, J. I. Climente, A. Prudnikau, A. Antanovich, R. Scott, M. V. Artemyev, J. Planelles and U. Woggon, J. Phys. Chem. C, 2015, 119, 1260-1267.

36 Y. Wang and N. Herron, J. Phys. Chem., 1991, 95, 525-532.

37 Y. Wang, X. Yang, T. C. He, Y. Gao, H. V. Demir, X. W. Sun and H. D. Sun, Appl. Phys. Lett., 2013, 102, 021917.

38 M. Allione, A. Ballester, H. B. Li, A. Comin, J. L. Movilla, J. I. Climente, L. Manna and I. Moreels, ACS Nano, 2013, 7, 2443-2452.

39 V. A. Fonoberov, E. P. Pokatilov and A. A. Balandin, Phys. Rev. B: Condens. Matter Mater. Phys., 2002, 66, 085310.

40 X. P. Li, J. Embden, J. W. M. Chon and M. Gu, Appl. Phys. Lett., 2009, 94, 103117.

41 J. He, J. Mi, H. P. Li and W. Ji, J. Phys. Chem. B, 2005, 109, 19184-19187.

42 A. W. Achtstein, J. Hennig, A. Prudnikau, M. V. Artemyev and U. Woggon, J. Phys. Chem. C, 2013, 117, 25756-25760.

43 J. He, W. Ji, G. H. Ma, S. H. Tang, E. S. W. Kong, S. Y. Chow, X. H. Zhang, Z. L. Hua and J. L. Shi, J. Phys. Chem. B, 2005, 109, 4373-4376.

44 M. Albota, D. Beljonne, J. L. Bredas, J. E. Ehrlich, J. Y. Fu, A. A. Heikal, S. E. Hess, T. Kogej, M. D. Levin, S. R. Marder, D. McCord-Maughon, J. W. Perry, H. Rockel, M. Rumi, C. Subramaniam, W. W. Webb, X. L. Wu and C. Xu, Science, 1998, 281, 1653-1656. 